\title{
Automated Processing of Multiple-Brightness Peak Histogram Image Using Curvature and Variance Estimation
}

\author{
Yusuke Kawakami $^{1}$, Tetsuo Hattori ${ }^{2}$, Yoshiro Imai ${ }^{2}$, Yo Horikawa ${ }^{2}$, Kazuaki Ando ${ }^{2}$, R. P. C. Janaka Rajapakse ${ }^{3}$ \\ ${ }^{1}$ DynaxT Co., Ltd. 2217-6 Hayashi \\ Takamatsu City, Kagawa 761-0301, Japan \\ E-mail: riverjp2002@gmail.com \\ ${ }^{2}$ Kagawa University, 2217-20 Hayashi \\ Takamatsu City, Kagawa 761-0396, Japan \\ E-mail: \{hattori, imai, horikawa, ando\}@eng.kagawa-u.ac.jp \\ ${ }^{3}$ Tainan National University of the Arts, 66 Daci \\ Guantian District, Tainan 72045, Taiwan \\ E-mail: janakaraja@gmail.com
}

\begin{abstract}
Previously, we have illustrated that the Histogram Matching based on Gaussian Distribution (HMGD) is an effective automated image processing method for obtaining a better feeling impression image. However, the simple HMGD works only for the image whose histogram has just one peak. For the image whose histogram has multiplebrightness peak, it does not work as in the case of single peak histogram image. In this paper, we propose the improved method for multiple-brightness peak (HMGD-MBP). This method can not only detect multiple peaks but also estimate the variance of Gaussian distribution at each detected peak in the image histogram, using curvature computation. This paper also presents the effectiveness of the proposed method by showing the experimental results.

Keywords: Image processing, Histogram, Curvature, Variance estimation, Histogram matching, HMGD
\end{abstract}

\section{Introduction}

Recently, automated image processing for the enhancement and arrangement of color images is getting popular in our familiar electronic equipment and computer systems, for example, Digital Signage, Smart Phone, etc. ${ }^{1-3}$

In the previous paper, we have presented that the Histogram Matching based on Gaussian Distribution (HMGD) processing is one of the effective automated image arrangement methods. ${ }^{4-5}$ And also, we have illustrated that HMGD processing works to obtain better feeling (or Kansei) impression than that of original image, by the comparative experimental investigation. ${ }^{6}$ However, HMGD processing has just worked only for single brightness-peak histogram image.

In this paper, we present the improved HMGD for multiple-brightness peak histogram image that we call "HMGD-MBP", using curvature computation and variance estimation. We explain the principle, and also give the procedure of peak detection and variance estimation of Gaussian distribution of each peak in the image histogram. Lastly, we show the effectiveness by illustrating the experimental results of the HMGDMBP. 


\section{Principle}

\subsection{Histogram Matching based on Gaussian Distribution (HMGD)}

In the section, we explain the principle of HMGD processing.

Fig. 1 shows the conceptual image of HMGD. Let $f(x)$ and $h(y)$ be two probabilistic density functions (PDF) on real variables $x$ and $y$, respectively. The $\mathrm{PDF}$ is corresponding to histogram of image brightness level which is discretely defined.

In addition, let $y=\phi(x)$ be a continuous and monotonic increase function corresponding to cumulative histogram of image brightness level between variables $x$ and $y .^{7-9}$ And let $\phi(x)$ be defined by Eq. (1).

$$
y=\phi(x)=L \int_{0}^{x} f(x) d x .
$$

First, we have to expand the brightness level of original image histogram and convert it into an uniform distribution histogram, because we aim to match Gaussian distribution. From Eq. (1) and Fig. 1, we can derive Eq. (2) and (3).

$$
\begin{gathered}
f(x) d x=h(y) d y=h(y) \phi^{\prime}(x) d x \\
=h(y) L f(x) d x . \\
h(y)=\frac{1}{L} .
\end{gathered}
$$

We understand the histogram of original image $f(x)$ becomes uniform distribution $h(y)$ by Eq. (3). It means that the distribution $f(x)$ is expanded to $h(y)$.

In the same way, let $\operatorname{Gauss}(z)$ and $\gamma(z)$ be two functions that are defined by Eq.(4) and Eq.(5), respectively.

$$
\begin{aligned}
& \operatorname{Gauss}(z)=\frac{1}{\sqrt{2 \pi \sigma^{2}}} \exp \left(-\frac{(z-\mu)^{2}}{2 \sigma^{2}}\right) . \\
& y=\gamma(z)=L \int_{0}^{z} \operatorname{Gauss}(z) d z \\
& =\frac{L}{\sqrt{2 \pi \sigma^{2}}} \int_{0}^{z} \exp \left(-\frac{(z-\mu)^{2}}{2 \sigma^{2}}\right) d z .
\end{aligned}
$$

From the relationship between $y=\phi(x)$ and $y=\chi(z)$ as shown in Fig. 1, we have the following Eq. (6).

$$
L \int_{0}^{x} f(x) d x=\frac{L}{\sqrt{2 \pi \sigma^{2}}} \int_{0}^{z} \exp \left(-\frac{(z-\mu)^{2}}{2 \sigma^{2}}\right) d z
$$

And we can derive Eq. (7) from Eq. (6).

$$
\frac{d}{d x} L \int_{0}^{x} f(x) d x=\frac{d}{d z}\left(\frac{L}{\sqrt{2 \pi \sigma^{2}}} \int_{0}^{z} \exp \left(-\frac{(z-\mu)^{2}}{2 \sigma^{2}}\right) d z\right) \frac{d z}{d x} .
$$

Then we have,

$$
f(x) d x=\operatorname{Gauss}(z) d z .
$$

That is, we understand that $f(x)$ is transformed into Gaussian distribution Gauss(z).

Thus, we can say that HMGD processing is a kind of a cumulative histogram transformation from the original histogram into Gaussian histogram. ${ }^{8}$

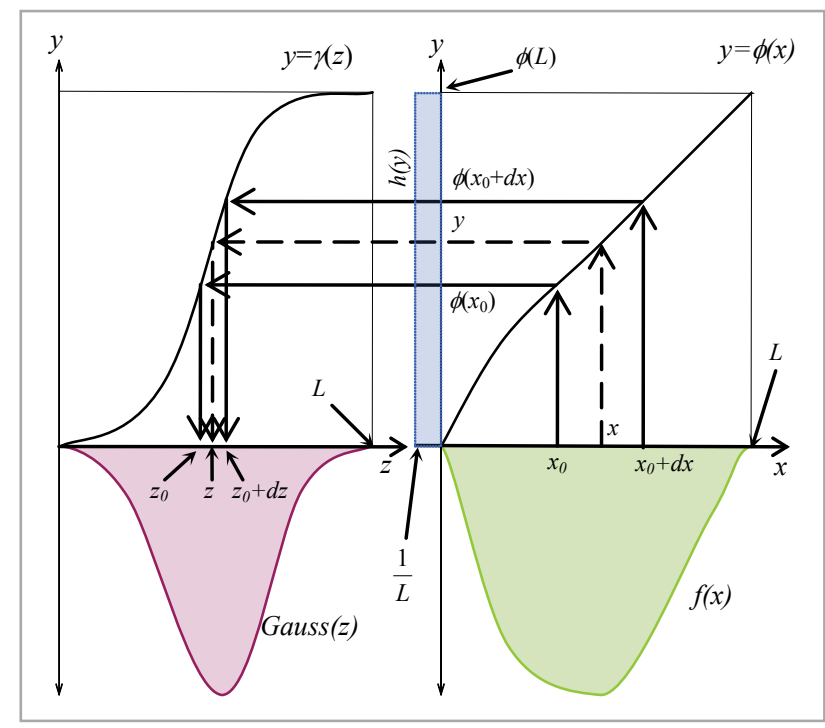

Fig. 1. Conceptual image of HMGD. ${ }^{6,8-10}$

\subsection{Peak Detection of Histogram in HMGD-MBP}

The HMGD processing method for multiple-brightness peak histogram image needs to detect peaks in image histogram. In order to do so, we use the curvature computation.

Let $y$ be a function with respect to $x$, the definition curvature $R$ is given by Eq. (9). ${ }^{6-10}$ 


$$
R=\frac{d^{2} y}{d x^{2}}\left(1+\left(\frac{d y}{d x}\right)^{2}\right)^{-\frac{3}{2}}
$$

Let $g(x)$ be a Gaussian distribution function as defined by the following equation, where $\mathrm{K}$ is a constant value.

$$
\begin{aligned}
& g(x)=\frac{K}{\sqrt{2 \pi \sigma^{2}}} \exp \left(-\frac{(x-a)^{2}}{2 \sigma^{2}}\right), \\
& \frac{K}{\sqrt{2 \pi \sigma^{2}}} \int_{0}^{L} \exp \left(-\frac{(u-a)^{2}}{2 \sigma^{2}}\right) d u=1 .
\end{aligned}
$$

Next, let $y=f(x)$ be a function representing the cumulative histogram which is represented Eq. (11). That is, $d y / d x$ and $d^{2} y / d x^{2}$ be described as Eq. (12) and Eq. (13), respectively. From Eq. (12) and Eq. (13), we obtain the approximation of curvature $R$ as Eq. (14).

$$
\begin{gathered}
f(x)=\int_{0}^{x} g(u) d u=\frac{K}{\sqrt{2 \pi \sigma^{2}}} \int_{0}^{x} \exp \left(-\frac{(u-a)^{2}}{2 \sigma^{2}}\right) d u . \\
\frac{d y}{d x}=g(x)=\frac{K}{\sqrt{2 \pi \sigma^{2}}} \exp \left(-\frac{(x-a)^{2}}{2 \sigma^{2}}\right) \\
\frac{d^{2} y}{d x^{2}}=\frac{d g(x)}{d x}=\frac{(a-x)}{\sigma^{2}} g(x) \\
R=\frac{\frac{(a-x)}{\sigma^{2}} g(x)}{\left(1+g(x)^{2}\right)^{\frac{3}{2}}} \approx \frac{(a-x)}{\sigma^{2}} g(x) .
\end{gathered}
$$

From Eq. (14), the curvature $R$ varies the sign according to $x$. That is, if $x<a \rightarrow R>0$ (downward convex shape), and if $x<a \rightarrow R<0$ (upward convex shape). ${ }^{9}, 10$

\subsection{Variance Estimation}

In this section, we propose how to optimize the shape of the reference histogram, which is used in the HMGD processing method for multiple-brightness peak image.

Fig. 2 shows the conceptual image of the original image histogram which is variance $\sigma^{2}$ and average $a$. And Fig. 3 shows its cumulative histogram. From Eq. (10), we have the following Eq. (15) and Eq. (16).

$$
\begin{aligned}
g(a) & =\frac{K}{\sqrt{2 \pi \sigma^{2}}}=\frac{K}{\sigma \sqrt{2 \pi}} . \\
g(a \pm \sqrt{2} \sigma) & =\frac{K}{\sigma \sqrt{2 \pi}} \exp \left(-\frac{(a \pm \sqrt{2} \sigma-a)^{2}}{2 \sigma^{2}}\right) \\
& =g(a) e^{-1} .
\end{aligned}
$$

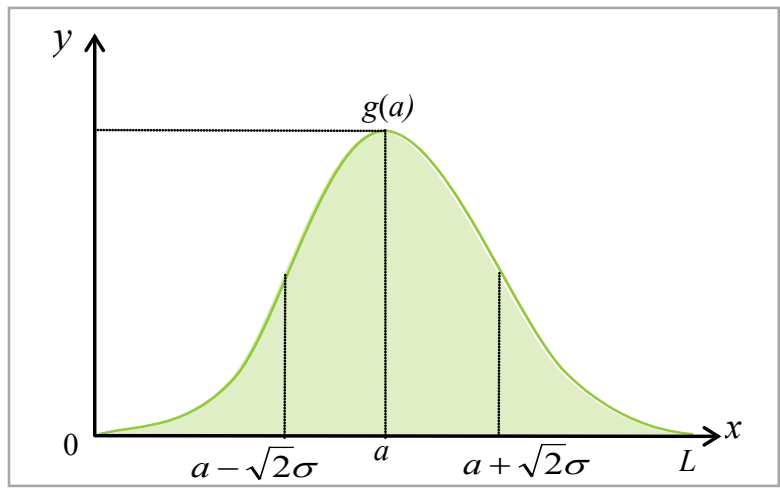

Fig. 2. Conceptual image of the original image histogram. ${ }^{8,10}$

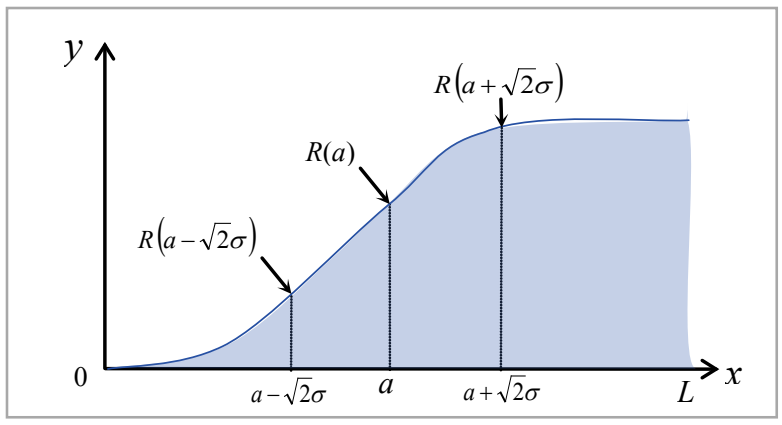

Fig. 3. Conceptual image of the original image cumulative histogram. ${ }^{8}$

Let $S=g(a) e^{-1}$. Then, from Eq.(14), the curvature $R$ at $x=a \pm \sqrt{2} \sigma$ is represented as follows.

$$
\begin{aligned}
& R(a \pm \sqrt{2} \sigma) \\
= & \left(\mp \frac{\sqrt{2}}{\sigma}\right) \frac{\left(g(a) e^{-1}\right)}{\left(1+\left(g(a) e^{-1}\right)^{2}\right)^{\frac{3}{2}}}=\left(\mp \frac{\sqrt{2}}{\sigma}\right) \frac{S}{\left(1+S^{2}\right)^{\frac{3}{2}}} .
\end{aligned}
$$

Then we have Eq. (18).

$$
R(a \pm \sqrt{2} \sigma)=\left(\mp \frac{\sqrt{2}}{\sigma}\right) H .
$$




$$
\text { where } \quad H=\frac{S}{\left(1+S^{2}\right)^{\frac{3}{2}}}=\frac{\left(g(a) e^{-1}\right)}{\left(1+\left(g(a) e^{-1}\right)^{2}\right)^{\frac{3}{2}}} \text {. }
$$

Let $v=\sqrt{2} \sigma$ be the distance from the average $a$, we have the following Eq. (20).

$$
R(a-v)-R(a+v)=\frac{4}{v} H
$$

Then, we can obtain the variance $\sigma^{2}$ from the curvature $R$ and peak value $g(a)$, using the following procedure.

(i) Compute the $H$ from the value $g(a)$ using Eq.(19).

(ii) Detect the $v$ that satisfies the Eq.(20).

(iii) Obtain the $\sigma^{2}=v^{2} / 2$.

\section{HMGD Processing for Multiple-Brightness Peak (HMGD-MBP)}

In this section, we present the concrete procedure of HMGD-MBP as follows.

(i) Detect the brightness-peak values $x_{m}(m=1, \ldots, n)$ of original image histogram (Fig. 4).

(ii) Perform variance estimation and generate the reference histogram for each brightness-peak value $x_{m}$.

(iii) Perform HMGD processing and make an image $i_{m}$ which has histogram $h_{m}$ (Fig. 5).

(iv) Proceed to (v) if the original image has no brightness-peak value. Otherwise, set the brightness-peak value to $x_{m+1}$ and return to (ii).

(v) Calculate the average of the HMGD processed (output) image $I_{a}$ using Eq. (21) (Fig. 6).

$$
I_{a}=\frac{1}{n} \sum_{m=1}^{n} i_{m}
$$

\section{Experimentation}

Fig.7 shows the example of results and the corresponding histograms by HMGD and HMGD-MBP, respectively.

In this case of Fig.7, we understand that HMGD-MBP processing is enhancing the contrast more naturally than HMGD. And also in the HMGD-MBP processed image, the edges in the mountain (located left below of image) becomes clear and detailed.
Fig.8 shows another example of results and the corresponding histogram for HMGD and HMGD-MBP, respectively.

In this case, we understand that HMGD-MBP processed image has more quiet color than original image. On the other hand, HMGD processed image is brighter than the original image. But its color becomes unnatural (located left side of image).

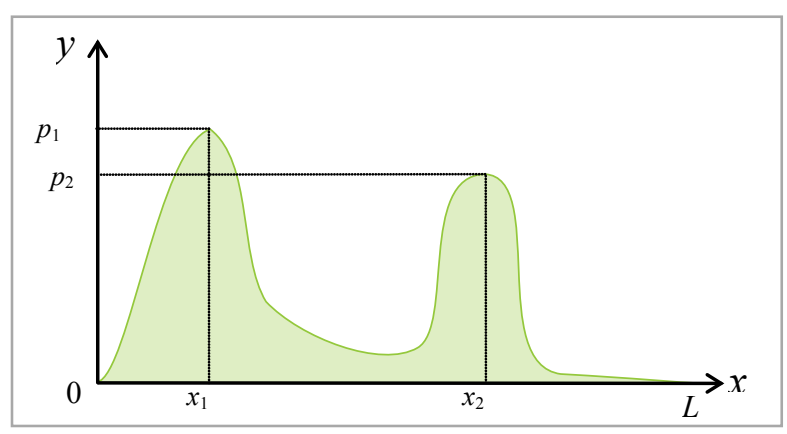

Fig. 4. Conceptual histogram of original image (multiplebrightness peak). ${ }^{9}$

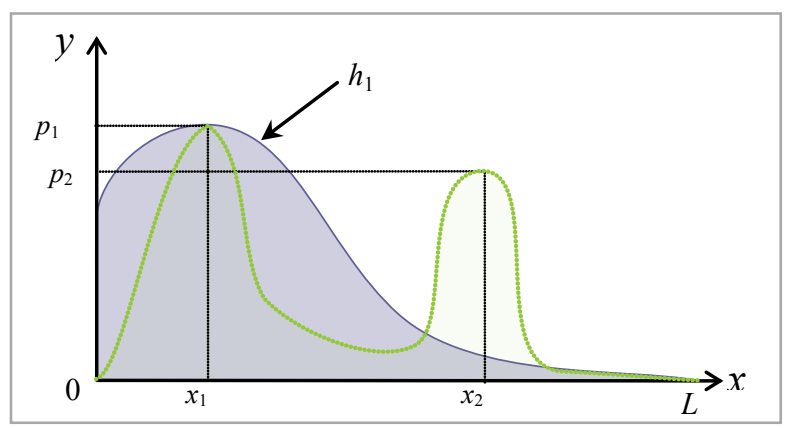

Fig. 5. Conceptual histogram of HMGD processed image (brightness peak $\left.x_{1}\right)^{9}$

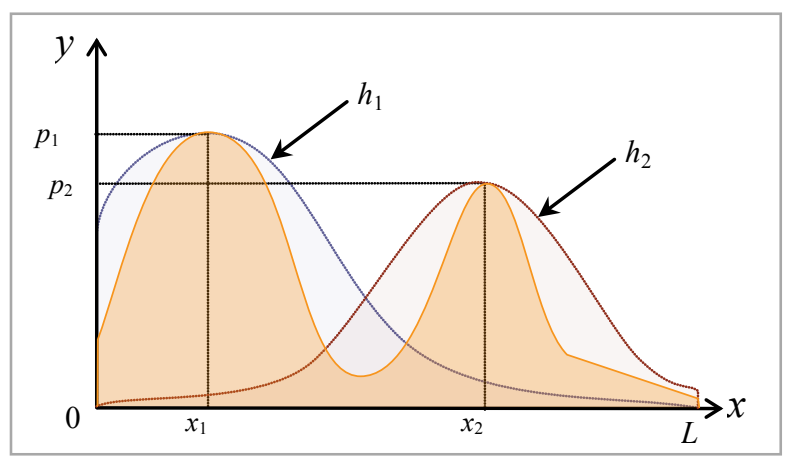

Fig. 6. Conceptual histogram of output image. ${ }^{9}$ 


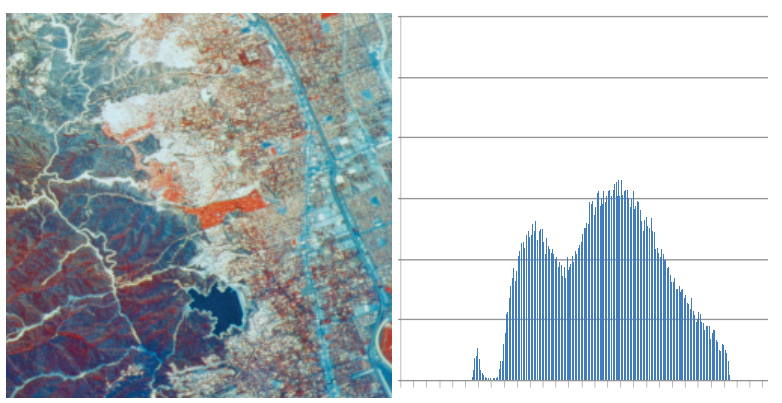

(a) Original image and its histogram

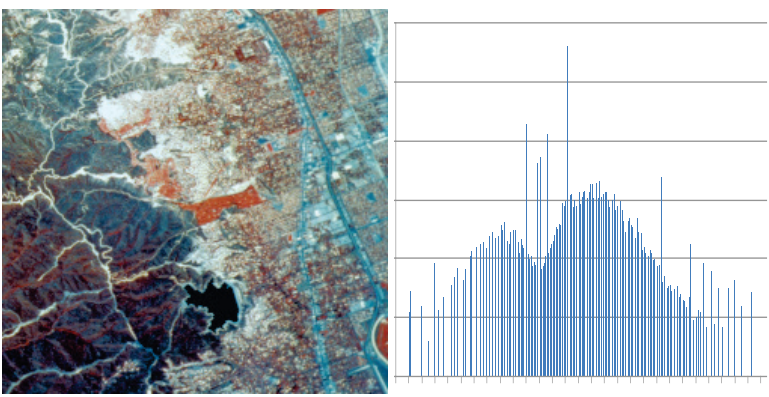

(b) HMGD processed image and its histogram

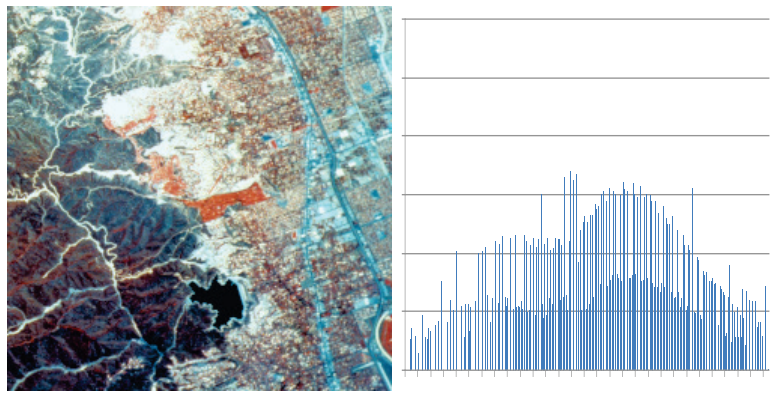

(c) HMGD-MBP processed image and its histogram

Fig. 7. Experimental results (I). ${ }^{10}$

\section{Conclusion}

In this paper, aiming to obtain an improved automated processing for multiple-brightness peak histogram image, we have proposed "Histogram Matching based on Gaussian Distribution for multiple-brightness peak histogram image" that we call "HMGD-MBP". As for the concrete processing method, we have used curvature computation for detecting brightness peaks and variance estimation of Gaussian distribution at each detected peak. After that, each of the peak shapes is transformed into Gaussian shape.

From the experimental results, the HMGD-MBP processing naturally enhances the contrast of image, and so the edges of image become clarified and detailed.
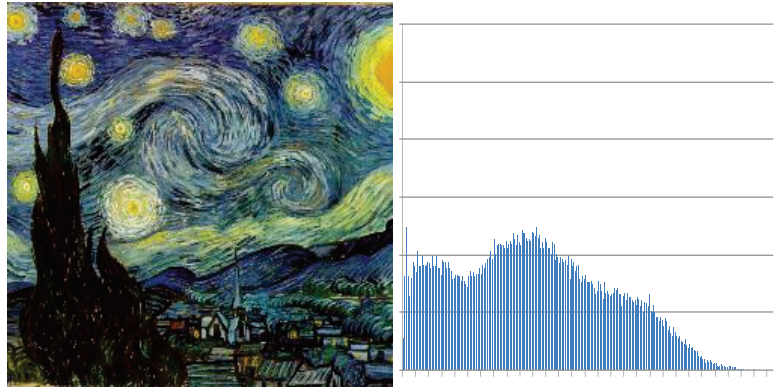

(a) Original image and its histogram

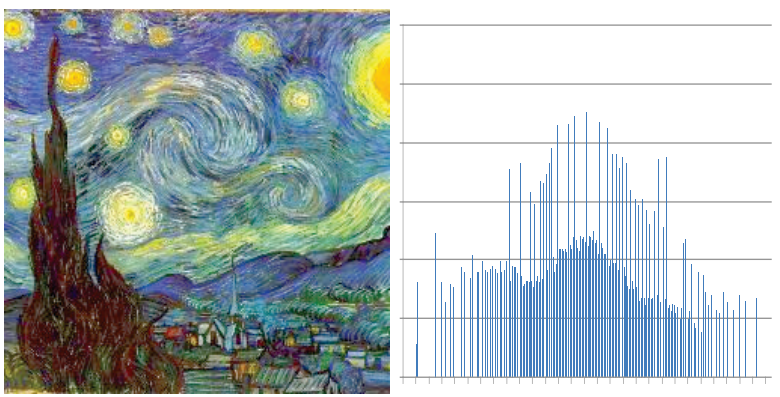

(b) HMGD processed image and its histogram

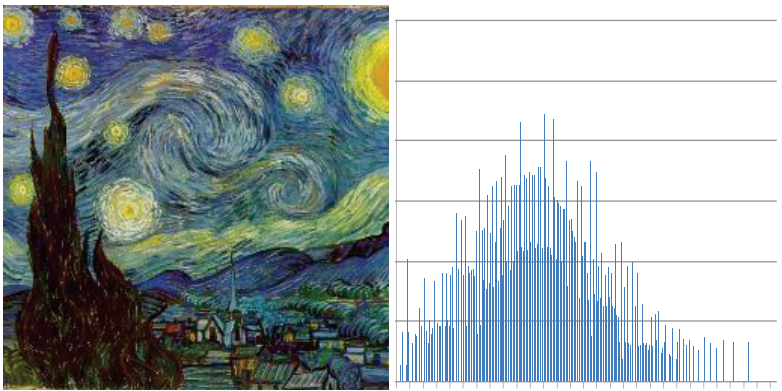

(c) HMGD-MBP processed image and its histogram

Fig. 8. Experimental results (II).

Also, we understand that the color of HMGD-MBP processed image tends to become more moderate than original image.As a conclusion, we consider that the HMGD-MBP processing method is more useful than HMGD.

For further study, we have to much more improve the proposed method so that it can arrange the colors and contrast of input image easily and in detail. In order for the image processing to have such arrangement capability, we would like to introduce some related processing methods of Signal Processing in Kansei Engineering, Time Series Analysis, and Advection Diffusion in Fluid Dynamics. ${ }^{11-15}$ 


\section{References}

1. R. C. Gonzalez and R. E. Woods, Digital Image Processing (Addison-Wesley Publishing Company, 1993).

2. B. Jahne, Digital Image Processing --Concepts, Algorithms, and Scientific Applications-- 4th edition (Springer, 1995).

3. E. S. Umbaugh, Computer Vision and Image Processing: A Practical Approach Using CVIP tools (Prentice Hall PTR, 1998).

4. W. Burger and J. M. Burge, Principles of Digital Image Processing: Fundamental Techniques (Springer 2009).

5. T. Izumi, T. Hattori, S. Sugimono, and T. Takashima, Color Image Arrangement Using Elastic Transform on Principal Component Axis (in Japanese), Journal of Japan Society of Kansei Engineering 8(3) (2009) 667674.

6. Y. Kawakami, T. Hattori, D. Kutsuna, H. Matsushita, Y. Imai, H. Kawano, R.P.C. Janaka Rajapakse, Automated Color Image Arrangement Method Based on Histogram Matching - Investigation of Kansei impression between HE and HMGD -, International Journal of Affective Engineering 14(2) (2015) ISSN 2187-5413, 8593.

7. Y. Kawakami, T. Hattori, Y. Imai, H. Matsushita, H. Kawano, R. P. C. Janaka Rajapakse, Kansei Impression and Automated Color Image Arrangement Method and Kansei Impression, Journal of Robotics, Networking and Artificial Life 1(1) (2014) ISSN 23526386, 60-67.

8. Y. Kawakami, T. Hattori, Y. Imai, H. Matsushita, H. Kawano, and R. P. C. Janaka Rajapakse, Automated Color Image Arrangement Method Using Curvature Computation in Histogram Matching, in Proceedings of International Conference on Artificial Life and Robotics (ICAROB 2015) (Oita, Japan, 2015) ISBN 978-49902880-9-9, pp. 272-277.
9. Y. Kawakami, T. Hattori, Y. Imai, Y. Horikawa, H. Matsushita, R. P. C. Janaka Rajapakse, Automated Color Image Arrangement Method for Multiple-Peak Image, Proceedings of The Second International Conference on Computer Science, Computer Engineering, \& Social Media (Lodz, Poland, 2015), ISBN 978-1-4799-1789-1, pp. 27-30.

10. Y. Kawakami, T. Hattori, Y. Imai, Y. Horikawa, R. P. C Janaka Rajapakse, Automated Multiple-Brightness Peak Image Processing Method Using Curvature and Variance Estimation, Proceedings of International Conference on Artificial Life and Robotics (ICAROB 2016) (Okinawa, Japan, 2016) ISBN 978-4-9908350-1-9, pp. 32-35.

11. Yusuke Kawakami, Tetsuo Hattori, Hiromichi Kawano, Tetsuya Izumi, Experimental Investigation of Feature Quantity in Sound Signal and Feeling Impression Using PCA, Journal of Robotics, Networking and Artificial Life 1(4), (2015), ISSN 2352-6386, pp. 303-311.

12. Yoshihide Koyama, Tetsuo Hattori, Hiromichi Kawano, Model Introduced SPRT for Structural Change Detection of Time Series (I) -- Formulation --, Journal of Robotics, Networking and Artificial Life 1(1) (2014) ISSN:23526386, pp. 54-59.

13. Yoshihide Koyama, Tetsuo Hattori, Katsunori Takeda, Hiromichi Kawano, Model Introduced SPRT for Structural Change Detection of Time Series (II) , Journal of Robotics, Networking and Artificial Life 1(3) (2014) ISSN:2352-6386, pp. 237-243.

14. Junichi Fujita, Daisuke Sato, Kensho Okamoto, Tetsuo Hattori, Novel Opt-coupling Transistor by LED and PD and its Application, Journal of Robotics, Networking and Artificial Life 1(3) (2014) ISSN:2352-6386, pp. 244-248.

15. Hiroki Sakamoto, Tetsuo Hattori, Akiomi Tada, Vanhoa Nguyen, Hiromichi Kawano, Analysis of Navier-Stokes Equation from the Viewpoint of Advection Diffusion, Journal of Robotics, Networking and Artificial Life 1(4) (2015) ISSN:2352-6386, pp. 265-269. 